\title{
Co-inheritance of PAX4 and BLK Mutations (MODY 7 and 9) in an 38 Year old African Patient with Ketosis-Prone Diabetes
}

${ }^{1}$ Gemeinschaftspraxis für Humangenetik \& Genetische Labore, Hamburg, Germ
${ }^{2}$ Schwerpunktpraxis, Zentrum für Diabetologie Bergedorf, Hamburg, Germany

\section{Introduction}

Ketosis-prone diabetes (KPD) is an emerging and uncommon form of diabetes characterized by fulminant diabetic ketoacidosis and phasic insulin dependence without any immunological autoantibody to islet antigens of classic type 1 diabetes. KPD is mostly observed in African-American populations. Multiple, severe forms of $\beta$-cell dysfunction appear to underlie the pathophysiology of KPD. The PAX4 gene, causing Maturity-onset diabetes of the young (MODY) subtype 9, already has been associated with ketosis-prone diabetes.

\section{Patient and Methods}

A case report of a 38-year old male African patient born in Nigeria with co-inheritance of PAX4 and BLK mutations (MODY 7 and 9) as a cause of ketosis prone diabetes is presented. The patient was hospitalized 2013 as an emergency with metabolic acidosis, partial respiratory compensation with hypocapnia and initial renal failure. Under intensive medical therapy (insulin) the metabolic situation could be normalized. MODY genes type 1-11 were analyzed by DNA-sequencing and MLPA analysis. No familial cases of diabetes are known. Further family members were not available for mutation analysis. Dec 2014 the patient moved to southern Germany.

Clinical Data before ketosis: IV/2012 - I/2013

Weight loss of $15 \mathrm{~kg}$ in the two month before admission, reduction of the general condition, thrush of the oral cavity

Clinical Data and Laboratory measurement at admission: I/2013

Weight: 81.1kg, BMI: 28,4 kg/m2, HbA1C: $>14 \%$, Autoantibodies negative: GAD < 0,1

IA2 < 0,10, Fasting Blood Glucose: $5.8 \mathrm{mmol} / \mathrm{l}(105 \mathrm{mg} / \mathrm{dl})$, Ketones: negative, Blood pressure: 100/70 mmHg, Chol: 135, HDL: 36, LDL: 70, TC: 142, Creatinine: 1,10; Monofilament: $3 / 3$

Clinical Data and treatment after remission : II/2013 - IV/2014

Jan 2013 - Dec 2014 total insulin dose per day: 38 IU; Feb 2014 Weight: 98.4kg; April 2014 Weight: $91.0 \mathrm{~kg}$; II/2013 HbA1C: 6,4\%; III/2013 HbA1C: 6,2\%; IV/2013 HbA1C:

7,0\%; Dec 2014 C-Peptid 1,95 and Fasting Blood Glucose $7 \mathrm{mmol} / \mathrm{L}(126 \mathrm{mg} / \mathrm{dl})$

\section{Results - Genetics}

DNA-Sequencing revealed two heterozygous mutations:

PAX4 gene, exon 1, PAX4,c.109C>T, p.(Arg37Trp); Ref. Seq.: NM_006193.2 BLK gene, exon 5, BLK,C.335T>C, p.(Phe112Ser); Ref. Seq.: NM_001715.2
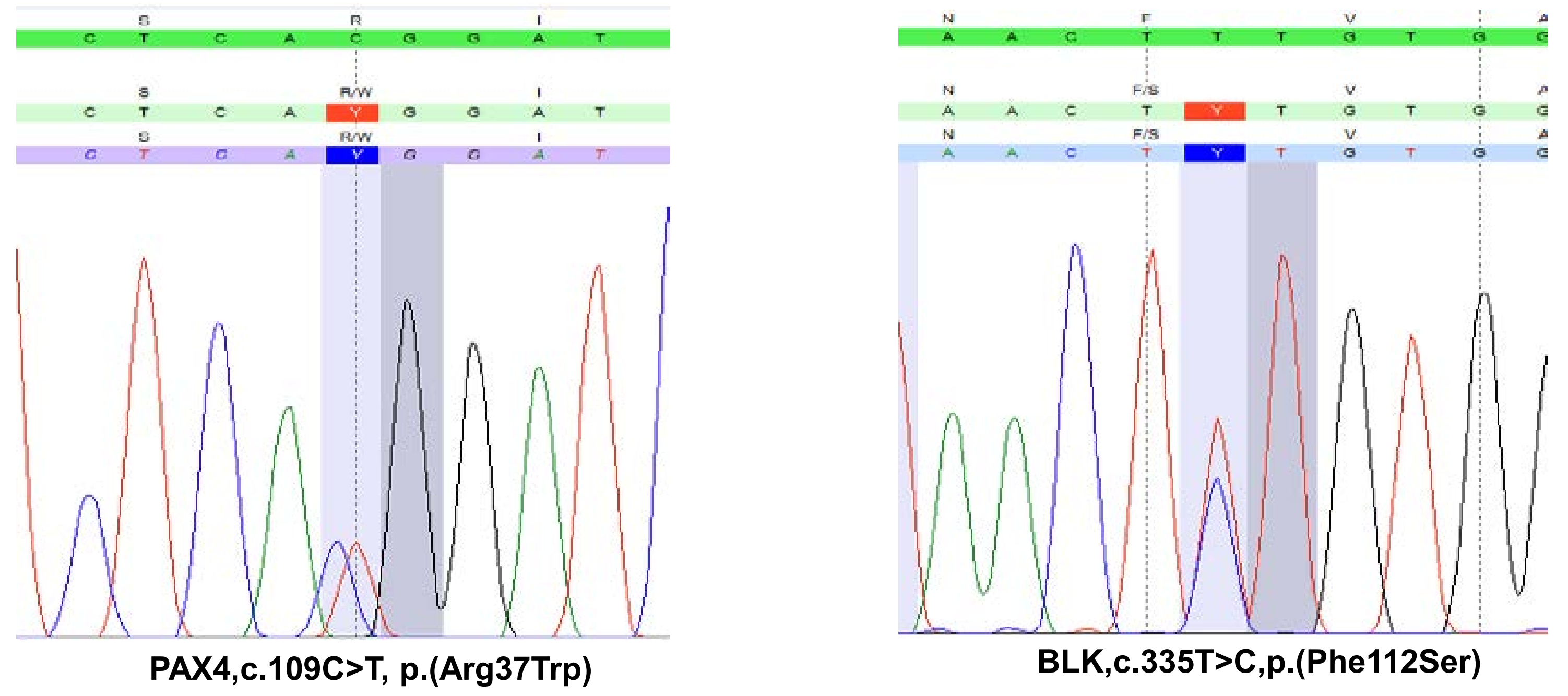

The amino acid position 37 in the PAX4 gene is located in an evolutionarily highly conserved sequence motif across many species boundaries and the amino acid position 112 in the BLK gene is located in the functional Srchomology $3(\mathrm{SH} 3)$ domain of the BLK protein.

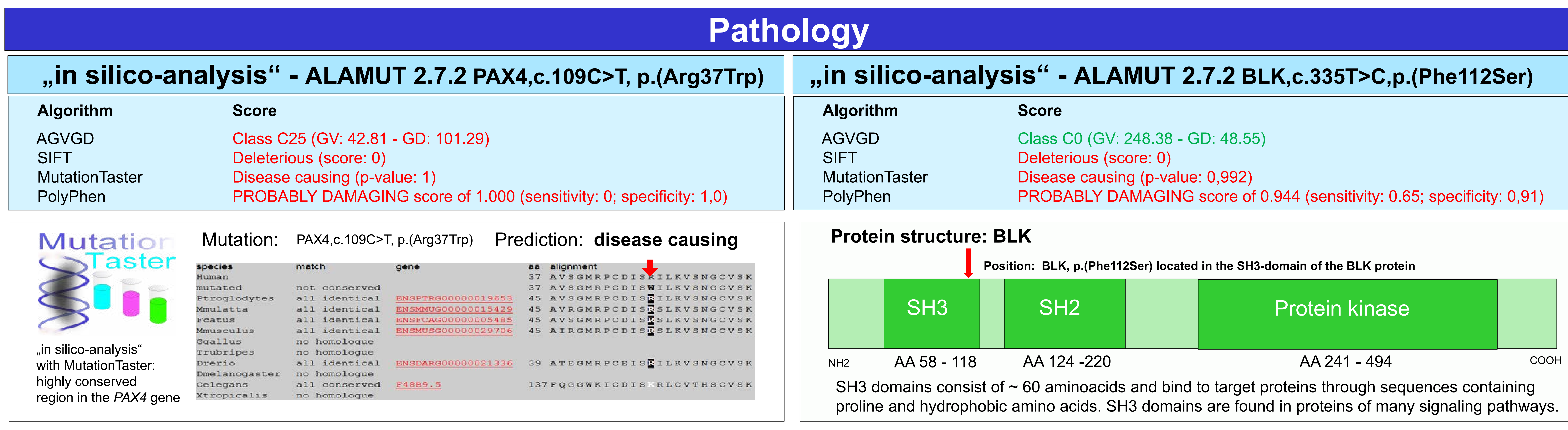

\section{Conclusion}

We identified two mutations in a heterozygous state: PAX4 gene, exon 1, PAX4,c.109C>T,p.(Arg37Trp) and BLK gene exon 5, BLK,c.335T>C, p.(Phe112Ser). Both mutations have been already described in the literature. Mutation PAX4,p.(Arg37Trp) has been found in a heterozygous state in one african patient from Cameroon with KPD. The authors could show that Arg37Trp affects the DNA-binding domain of the molecule and in vitro shows a $50 \%$ reduction of PAX4-binding activity to target gene promoters resulting in a similar decrease of normal PAX4 transcriptional repression activity (1). Here we describe this mutation for the second time in an african patient from Nigeria with KPD. The second mutation BLK,p.(Phe112Ser) has been associated with hypertriglyceridaemia (2) but triglycerides in our patient were normal at admission. Amino acid position 112 in the BLK gene is located in the functional SH3-domain of the BLK protein. "in-silico Analysis" of the mutation BLK,c.335T>C, p.(Phe112Ser) using the mutation analysis software ALAMUT (version 2.7.2) shows a high probability for a clinical relevance.

Co-inheritance of both mutations was never published before. Both mutations are located in evolutionarily highly conserved sequence motifs across many species boundaries indicating that they are highly likely pathogenic mutations. Further functional protein studies are needed to confirm PAX4 and BLK mutational analysis as a useful tool in clinical practice of KPD. Identification of the underlying genetic causes of KPD will give a better view of the mechanisms that contribute to the pathophysiology of the disease. Furthermore, proper identification may improve options to prevent KPD.

\section{Literature}

(1) PAX4 gene variations predispose to ketosis-prone diabetes. (Mauvais-Jarvis (2004) Hum Mol Genet 13:)

(2) LipidSeq: a next-generation clinical resequencing panel for monogenic dyslipidemias. (Johansen (2014) J Lipid Res 55: 765)

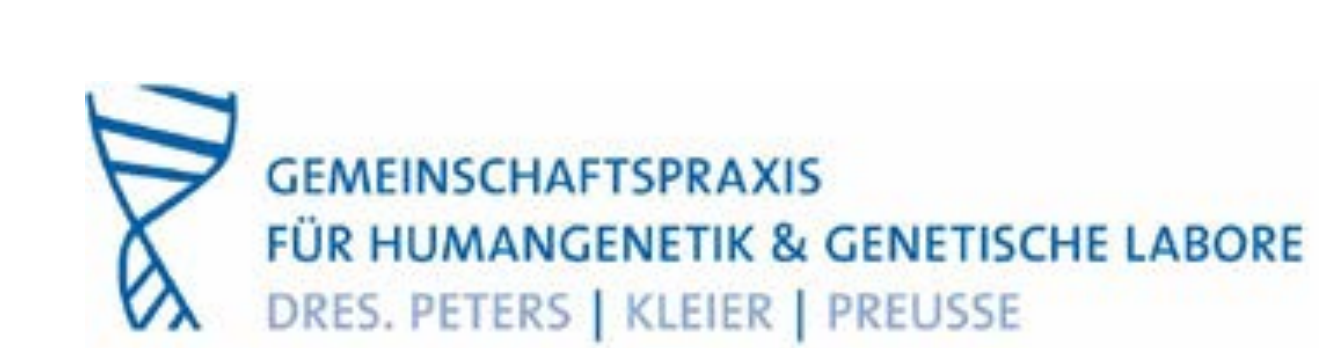

